\title{
Interferometric sensitivity and entanglement by scanning through quantum phase transitions in spinor Bose-Einstein condensates
}

\author{
P. Feldmann, ${ }^{1}$ M. Gessner, ${ }^{2}$ M. Gabbrielli,,${ }^{2,3}$ C. Klempt, ${ }^{4}$ L. Santos,,${ }^{1}$ L. Pezzè,${ }^{2}$ and A. Smerzi ${ }^{2}$ \\ ${ }^{1}$ Institut für Theoretische Physik, Leibniz Universität Hannover, Appelstraße 2, DE-30167 Hannover, Germany \\ ${ }^{2}$ QSTAR, INO-CNR, and LENS, Largo Enrico Fermi 2, IT-50125 Firenze, Italy \\ ${ }^{3}$ Dipartimento di Fisica e Astronomia, Università degli Studi di Firenze, via Sansone 1, I-50019 Sesto Fiorentino, Italy \\ ${ }^{4}$ Institut für Quantenoptik, Leibniz Universität Hannover, Welfengarten 1, DE-30167 Hannover, Germany
}

(Received 22 December 2017; published 27 March 2018)

\begin{abstract}
Recent experiments demonstrated the generation of entanglement by quasiadiabatically driving through quantum phase transitions of a ferromagnetic spin-1 Bose-Einstein condensate in the presence of a tunable quadratic Zeeman shift. We analyze, in terms of the Fisher information, the interferometric value of the entanglement accessible by this approach. In addition to the Twin-Fock phase studied experimentally, we unveil a second regime, in the broken axisymmetry phase, which provides Heisenberg scaling of the quantum Fisher information and can be reached on shorter time scales. We identify optimal unitary transformations and an experimentally feasible optimal measurement prescription that maximize the interferometric sensitivity. We further ascertain that the Fisher information is robust with respect to nonadiabaticity and measurement noise. Finally, we show that the quasiadiabatic entanglement preparation schemes admit higher sensitivities than dynamical methods based on fast quenches.
\end{abstract}

DOI: 10.1103/PhysRevA.97.032339

\section{INTRODUCTION}

Atom interferometry has become an indispensable tool for both the testing of fundamental physics and precision measurements [1]. Without entanglement between the atoms, the attainable sensitivity is fundamentally limited by the standard quantum limit (SQL) [2,3]. Employing multipartite entanglement allows to shift this bound towards the Heisenberg limit (HL) [2-4]. In view of the high effort required for handling coherent ensembles with a large atom number $N$, it is crucial that the HL replaces the SQL scaling of the sensitivity $\propto \sqrt{N}$ with $\propto N$. Entanglement that, in the absence of technical noise, facilitates to surpass the SQL is unambiguously witnessed by the Fisher information (FI).

In spinor Bose-Einstein condensates (BECs), entanglement useful to enhance the sensitivity of atom interferometers beyond the SQL can be generated exploiting spin-changing collisions [5]. A common realization relies on the parametric amplification of quantum fluctuations leading to squeezed Gaussian states [6-12]. Sub-SQL sensitivities [6,11,12], entanglement $[9,10]$, and squeezing of up to $10 \mathrm{~dB}$ beyond the SQL $[7,8,10]$ have been demonstrated [5]. Furthermore, multipartite entanglement in spinor BECs can also be generated near the ground state of ferromagnetic [13] and antiferromegnetic [14] spin-1 BECs [15]. In the following we focus on the ferromagnetic case, relevant for experiments with ${ }^{87} \mathrm{Rb}$. In the presence of an (effective) quadratic Zeeman shift $q$, the system exhibits three quantum phases $[13,16]$. By preparing the BEC in the ground state at $q>q_{c}$ (here $q_{c}>0$ and the critical points are located at $q= \pm q_{c}$ ) and slowly driving through both quantum phase transitions an entanglement depth of about 900 particles has been witnessed in the Twin-Fock (TF) phase at $q<-q_{c}$ [17], see also [18,19].
Recently [20], we showed that the ground state of a ferromagnetic BEC at $q=0$ can be used for heralded generation of highly entangled macroscopic superposition states. In the present paper we extend this study and analyze the interferometric sensitivity of the entangled quantum states that are generated along the (quasi)adiabatic passage when scanning over different values of $q$. Their full potential is revealed by considering atom interferometry involving all three modes, which generalizes the two-mode interferometry experimentally implemented, e. g., in [11]. We find that besides the TF state studied in $[13,17]$ also the ground state at the center, $q=0$, of the broken axisymmetry phase leads to Heisenberg scaling. This state can be reached by (quasi)adiabatically scanning over only a single critical point, stopping the evolution half-way to the TF state. We identify the interferometric transformations that provide the most sensitive phase imprinting and demonstrate that the measurement of particle numbers, an established experimental technique, is optimal for the phase estimation. Our simulations show that performing the passage within reasonable, finite time does not strongly impair the attainable FI. We further analyze the effect of measurement noise and find that surpassing the SQL with state-of-the-art technology is well feasible. We finally show that, under realistic conditions, quasiadiabatic schemes produce states with larger interferometric sensitivity than those accessible by parametric amplification.

\section{FISHER INFORMATION AND INTERFEROMETRY}

Let us briefly review some concepts used in the paper. In any atom interferometer, a phase $\theta$ is imprinted into an initial density matrix $\hat{\rho}_{0}$, leading to a $\hat{\rho}_{\theta}$ which is subsequently measured to determine the phase $\theta$. The resulting estimation 
of $\theta$ has an uncertainty, which is bounded by the (classical) Cramér-Rao bound $\Delta \theta \geqslant \Delta \theta_{\mathrm{CR}}$. Here

$$
\Delta \theta_{\mathrm{CR}}=1 / \sqrt{v F}
$$

and

$$
F(\theta)=\sum_{\mu} \frac{1}{P(\mu \mid \theta)}\left(\frac{\partial P(\mu \mid \theta)}{\partial \theta}\right)^{2}
$$

is the (classical) FI which depends on $\hat{\rho}_{\theta}$ and the chosen measurement observable. The sum comprises all possible measurement outcomes $\mu$ and $P(\mu \mid \theta)$ is the probability to measure $\mu$ given that the quantum state is $\hat{\rho}_{\theta}$. Finally, $v$ is the number of measurements [21]. Maximizing the FI over all possible generalized quantum measurements defines the quantum Fisher information $(\mathrm{QFI}) F_{Q}$ [21-23]: $F \leqslant F_{Q}$ and the equality $F=F_{Q}$ can always be reached by an optimal measurement [22]. Correspondingly, a quantum Cramér-Rao bound is introduced as

$$
\Delta \theta_{\mathrm{QCR}}=1 / \sqrt{\nu F_{Q}}
$$

with $\Delta \theta_{\mathrm{CR}} \geqslant \Delta \theta_{\mathrm{QCR}}$. For $N$ qubits, we have $F_{Q} \leqslant N^{2}$ (HL) [2,3], and $F_{Q} \leqslant N$ (SQL) if $\hat{\rho}_{0}$ is not entangled [3]. Thus both the classical and quantum FI witness interferometrically useful entanglement: $F_{Q} \geqslant F>N$ is equivalent to a $\Delta \theta_{\mathrm{QCR}}$ undercutting the SQL.

We assume that the phase $\theta$ is imprinted by a collective unitary transformation across $n$ modes. Let $\hat{g}_{j}$ be the generators of the defining representation of $\mathrm{SU}(n)$. We denote the vector comprising these $d_{n}=n^{2}-1$ generators with respect to the $i$ th of $N$ particles as $\hat{\mathbf{g}}^{(i)} \equiv\left(\hat{g}_{1}^{(i)}, \ldots, \hat{g}_{d_{n}}^{(i)}\right)$. Then the final density matrix acquires the form $\hat{\rho}_{\theta}=\hat{U}(\theta) \hat{\rho}_{0} \hat{U}^{\dagger}(\theta)$, with $\hat{U}(\theta)=$ $\exp (-i \theta \mathbf{u} \cdot \hat{\mathbf{G}})$, where we call $\hat{\mathbf{G}}=\sum_{i=1}^{N} \hat{\mathbf{g}}^{(i)}$ a collective transformation, and $\mathbf{u} \in S^{d_{n}-1}$ is the interferometric direction. For a pure initial state, $\hat{\rho}_{0}=|\psi\rangle\langle\psi|$, the QFI due to an interferometric transformation generated by $\hat{R}_{\mathbf{u}} \equiv \mathbf{u} \cdot \hat{\mathbf{G}}$ reads

$$
F_{Q}\left[|\psi\rangle, \hat{R}_{\mathbf{u}}\right]=4\left(\Delta \hat{R}_{\mathbf{u}}\right)^{2}=4 \mathbf{u}^{T} \boldsymbol{\Gamma}_{\hat{\mathbf{G}}} \mathbf{u},
$$

where $\boldsymbol{\Gamma}_{\hat{\mathbf{G}}}$ denotes the covariance matrix of the operators composing $\hat{\mathbf{G}}$ [24], with elements $\left(\boldsymbol{\Gamma}_{\hat{\mathbf{G}}}\right)_{i j}=\left\langle\hat{G}_{i} \hat{G}_{j}\right\rangle / 2+$ $\left\langle\hat{G}_{j} \hat{G}_{i}\right\rangle / 2-\left\langle\hat{G}_{i}\right\rangle\left\langle\hat{G}_{j}\right\rangle$. The leading eigenvector of $\boldsymbol{\Gamma}_{\hat{\mathbf{G}}}$ identifies the optimal interferometric direction $\mathbf{u}_{\text {opt }}$. By convention, in the case of qubits $(n=2)$ the $\hat{g}_{i}$ are normalized such that $\left(\gamma_{i \max }-\gamma_{i \min }\right)^{2}=1, \gamma_{i \max }$ and $\gamma_{i \text { min }}$ being the maximum and minimum eigenvalues of $\hat{g}_{i}$, respectively. More generally, the SQL is given by $\left(\gamma_{i \max }-\gamma_{i \text { min }}\right)^{2} N$ and the HL by $\left(\gamma_{i \max }-\right.$ $\left.\gamma_{i \min }\right)^{2} N^{2}[2]$.

\section{MODEL}

In the following we study an optically trapped spin-1 BEC of $N$ particles with magnetic sublevels $m_{f} \in\{0, \pm 1\}$. In the single-mode approximation, the spinor dynamics is modeled by the Hamiltonian $[25,26]$

$\hat{H}=\left[\lambda\left(\hat{N}_{0}-\frac{1}{2}\right)+q\right]\left(\hat{N}_{+}+\hat{N}_{-}\right)+\lambda\left(\hat{a}_{1}^{\dagger} \hat{a}_{-1}^{\dagger} \hat{a}_{0}^{2}+\hat{a}_{0}^{\dagger 2} \hat{a}_{1} \hat{a}_{-1}\right)$, where $\hat{a}_{i}^{\dagger}$ and $\hat{a}_{i}$ are the creation and annihilation operators for $m_{f}=i$, and $\hat{N}_{0, \pm}=\hat{a}_{0, \pm 1}^{\dagger} \hat{a}_{0, \pm 1}$ are the number operators for the respective sublevels. The total number of atoms is equal to $N$ and is assumed fixed here. The interaction coefficient $\lambda$ (negative for the considered case of ferromagnetic condensates such as the $F=1$ hyperfine ground-state manifold of ${ }^{87} \mathrm{Rb}$ ) depends on the trapping potential and microscopic parameters, namely the scattering lengths and the mass of the atoms $[26,27]$. The effective quadratic Zeeman shift $q$ may be controlled by an external magnetic field and near-resonant microwave dressing $[16,26]$. Spin-changing collisions, described by the last line of Eq. (5), preserve the total magnetization, i. e., the eigenvalue $D$ of $\hat{D} \equiv \hat{N}_{+}-\hat{N}_{-}$. Hence starting from an initial condensate in $m_{f}=0$ and then quenching or slowly driving the magnetic field so to prepare entangled states ensures that the system remains in the subspace of $D=0$. The dynamics thus takes place in the Hilbert space spanned by the Fock states $|k\rangle \equiv$ $\left|N_{-}=k, N_{0}=N-2 k, N_{+}=k\right\rangle$ with $N_{i}$ the eigenvalues of $\hat{N}_{i}$. By restricting the dynamics to the magnetization-free subspace, the linear coupling to the magnetic field and its fluctuations (linear Zeeman shift) becomes irrelevant, which leads to phase noise stability.

In the magnetization-free subspace, model (5) presents three quantum phases $[13,16]$ as a function of $q$ with quantum phase transitions at $q= \pm q_{c}, q_{c}=2 N|\lambda|$ : the polar $(\mathrm{P})$ phase $(q>$ $\left.q_{c}\right)$, the broken-axisymmetry (BA) phase $\left(|q|<q_{c}\right)$, and the TF phase $\left(q<-q_{c}\right)$. For large $N$, the respective ground states approach $|k=0\rangle$ in the $\mathrm{P}$ phase and the TF state $|\mathrm{TF}\rangle \equiv \mid k=$ $N / 2\rangle$ in the TF phase. In the BA phase, all the three modes stay populated, with an average number of particles in $m_{f}=0$ given by $\left\langle\hat{N}_{0}\right\rangle / N \simeq\left(1+q / q_{c}\right) / 2[5]$.

\section{USEFUL ENTANGLEMENT IN THE GROUND STATE OF A SPINOR BEC}

We first evaluate the QFI of the ground state $\left|\psi_{0}(q)\right\rangle$ of the Hamiltonian (5) in the different phases. Arbitrary collective unitary rotations of a system of indistinguishable spin-1 particles, as considered in this paper, can be expressed by taking $\hat{\mathbf{G}}$ as the 8-dimensional vector of collective Gell-Mann operators, generating the $\mathrm{SU}(3)$. The covariance matrix $\Gamma_{\hat{\mathbf{G}}}$ is discussed in Appendix A 1. We find it convenient to introduce the symmetric $(g)$ and antisymmetric $(h)$ creation and annihilation operators

$$
\hat{g}^{\dagger}=\frac{1}{\sqrt{2}}\left(\hat{a}_{1}^{\dagger}+\hat{a}_{-1}^{\dagger}\right), \quad \hat{h}^{\dagger}=\frac{1}{\sqrt{2}}\left(\hat{a}_{1}^{\dagger}-\hat{a}_{-1}^{\dagger}\right)
$$

and present our results in terms of three sets of collective pseudospin-1/2 operators, whose Schwinger representation reads

$$
\begin{array}{ll}
\hat{S}_{x}=\frac{\hat{a}_{0}^{\dagger} \hat{g}+\hat{g}^{\dagger} \hat{a}_{0}}{2}, & \hat{J}_{x}=\frac{\hat{a}_{1}^{\dagger} \hat{a}_{-1}+\hat{a}_{-1}^{\dagger} \hat{a}_{1}}{2}, \\
\hat{S}_{y}=\frac{\hat{a}_{0}^{\dagger} \hat{g}-\hat{g}^{\dagger} \hat{a}_{0}}{2 i}, & \hat{J}_{y}=\frac{\hat{a}_{1}^{\dagger} \hat{a}_{-1}-\hat{a}_{-1}^{\dagger} \hat{a}_{1}}{2 i}, \\
\hat{S}_{z}=\frac{\hat{a}_{0}^{\dagger} \hat{a}_{0}-\hat{g}^{\dagger} \hat{g}}{2}, & \hat{J}_{z}=\frac{\hat{a}_{1}^{\dagger} \hat{a}_{1}-\hat{a}_{-1}^{\dagger} \hat{a}_{-1}}{2},
\end{array}
$$

and $\hat{A}_{i}$ just as $\hat{S}_{i}$ with $\hat{g}$ replaced by $\hat{h}$. Thus $\hat{\mathbf{S}} \equiv\left(\hat{S}_{1}, \hat{S}_{2}, \hat{S}_{3}\right)$ generates rotations within the two-level system composed of 


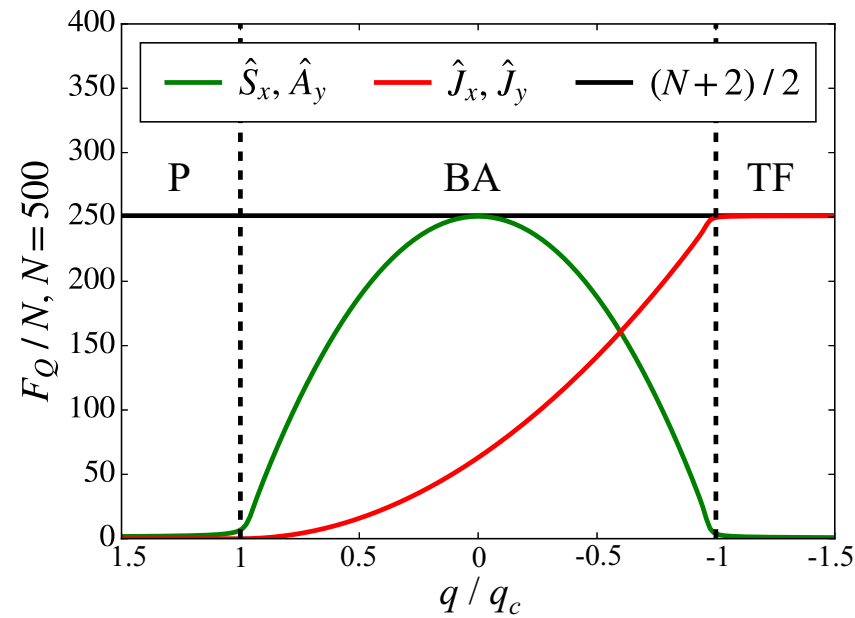

FIG. 1. QFI for the ground state of a nonmagnetized ferromagnetic spin-1 BEC as a function of the quadratic Zeeman shift $q$. For $\hat{\mathbf{G}}$ generating the $\mathrm{SU}(3)$, we depict $F_{Q} / N$ corresponding to the eigendirections of $\boldsymbol{\Gamma}_{\hat{\mathbf{G}}}$ for which $F_{Q}$ exceeds $N$. The quantum phase transitions are indicated by dashed vertical lines. The solid horizontal line is $F_{Q} / N=(N+2) / 2$.

the modes $\left(\hat{a}_{0}, \hat{g}\right), \hat{\mathbf{A}} \equiv\left(\hat{A}_{1}, \hat{A}_{2}, \hat{A}_{3}\right)$ corresponds to $\left(\hat{a}_{0}, \hat{h}\right)$, and $\hat{\mathbf{J}} \equiv\left(\hat{J}_{x}, \hat{J}_{y}, \hat{J}_{z}\right)$ to $\left(\hat{a}_{1}, \hat{a}_{-1}\right)$. Figure 1 displays, across the three quantum phases $\mathrm{P}, \mathrm{BA}$, and $\mathrm{TF}, F_{Q} / N$ corresponding to the eigenvectors of $\Gamma_{\hat{\mathbf{G}}}$ which provide $F_{Q}>N$. Large values of the QFI are observed in two cases. First, in the TF phase,

$$
F_{Q}\left[|\mathrm{TF}\rangle, \hat{R}_{\mathrm{opt}}^{(\mathrm{TF})}\right]=N(N+2) / 2,
$$

where $\hat{R}_{\mathrm{opt}}^{(\mathrm{TF})}$ is given by an arbitrary linear combination of $\hat{J}_{x}$ and $\hat{J}_{y}$. Second, at the center of the BA phase (i. e., for $q=0$, we indicate as $|\mathrm{CBA}\rangle$ the corresponding ground state) we have

$$
F_{Q}\left[|\mathrm{CBA}\rangle, \hat{R}_{\mathrm{opt}}^{(\mathrm{CBA})}\right]=N(N+1) / 2,
$$

where $\hat{R}_{\mathrm{opt}}^{(\mathrm{CBA})}$ is an arbitrary linear combination of $\hat{S}_{x}$ and $\hat{A}_{y}$. As we show in Appendix A2, the state has an explicit expression given by

$$
|\mathrm{CBA}\rangle \equiv \sqrt{\frac{2^{N}(N !)^{3}}{(2 N) !}} \sum_{k=0}^{\lfloor N / 2\rfloor} \frac{1}{2^{k} k ! \sqrt{(N-2 k) !}}|k\rangle .
$$

Hence both $|\mathrm{CBA}\rangle$ and $|\mathrm{TF}\rangle$ present approximately equal QFI and a Heisenberg scaling $F_{Q} \propto N^{2}$. It is well known that the ground state of the TF phase approaches a TF state [13] and that the latter exhibits a QFI with Heisenberg scaling with $N$ $[5,6,28]$. For an analysis of the QFI of the ground state of an antiferromagnetic spin-1 BEC see [14]. Conversely, Eq. (9) is a novel and less evident result. To gain some intuition regarding the large amount of useful entanglement found in the BA phase at $q=0$, let us rewrite the Hamiltonian (5) in terms of the $\hat{\mathbf{S}}$ and $\hat{\mathbf{A}}$ operator manifolds of Eq. (7). We obtain, up to c-numbers

$$
\hat{H}=2\left(\lambda \hat{S}_{x}^{2}-\frac{q}{3} \hat{S}_{z}\right)+2\left(\lambda \hat{A}_{y}^{2}-\frac{q}{3} \hat{A}_{z}\right),
$$

which is a sum of two (noncommuting) Lipkin-Meshkov-Glick Hamiltonians for $\hat{\mathbf{S}}$ and $\hat{\mathbf{A}}$, respectively. Since $\lambda<0$, the ground state of the first term in Eq. (11), $2\left(\lambda \hat{S}_{x}^{2}-\frac{q}{3} \hat{S}_{z}\right)$, at $q=0$
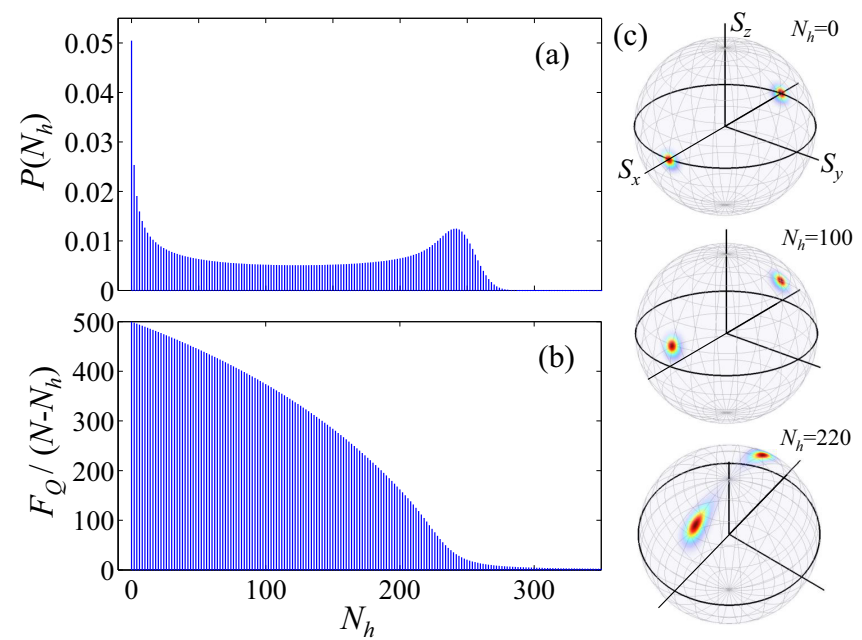

FIG. 2. (a) Probability $P\left(N_{h}\right)$ to find $N_{h}$ particles in the $h$ mode of $|\mathrm{CBA}\rangle$. (b) QFI $F_{Q}\left[\left|\phi_{N_{h}}\right\rangle, \hat{S}_{x}\right]$ of the states $\left|\phi_{N_{h}}\right\rangle$ obtained by projecting $|\mathrm{CBA}\rangle$ onto a fixed number of particles $N_{h}$ in the $h$ mode. (c) Husimi distributions of $\left|\phi_{N_{h}}\right\rangle$ for different values of $N_{h}$ showing the presence of NOON-like states in a very broad range of $N_{h}$ values. In this Figure $N=500$.

is a NOON state aligned along the $S_{x}$ axis (i. e., a superposition of the maximum and the minimum eigenstates of $\hat{S}_{x}$ ). Its QFI saturates the Heisenberg limit for rotations generated by $\hat{S}_{x}$. Similarly, the ground state of the second term of Eq. (11), at $q=0$, is a NOON state aligned along the $A_{y}$ axis. This hints at large amounts of entanglement in the CBA state. However, since the symmetric and antisymmetric spin algebras share the same central mode $\hat{a}_{0}$ and therefore do not commute with each other, a more detailed inspection of the ground state is required. To this end, let us trace out the $\hat{h}$ mode. This leaves us with the state

$$
\hat{\rho}=\sum_{N_{h}=0}^{N} P\left(N_{h}\right)\left|\phi_{N_{h}}\right\rangle\left\langle\phi_{N_{h}}\right|,
$$

in the modes $\left(\hat{a}_{0}, \hat{g}\right)$, where $P\left(N_{h}\right)$ is the probability to measure $N_{h}$ particles in the $\hat{h}$ mode, and $\left|\phi_{N_{h}}\right\rangle$ is a state of $N-N_{h}$ particles in $\left(\hat{a}_{0}, \hat{g}\right)$. In Fig. 2(a) we plot $P\left(N_{h}\right)$ as a function of $N_{h}$. The most probable value of $N_{h}$ is $N_{h}=0$, and $P\left(N_{h}\right)=0$ for odd values of $N_{h}$. Since $\hat{S}_{x}$ commutes with $\hat{N}_{h} \equiv \hat{h}^{\dagger} \hat{h}$ and $\left\langle\mathrm{CBA}\left|\hat{S}_{x}\right| \mathrm{CBA}\right\rangle=0$, the QFI decomposes according to

$$
F_{Q}\left[|\mathrm{CBA}\rangle, \hat{S}_{x}\right]=\sum_{N_{h}=0}^{N} P\left(N_{h}\right) F_{Q}\left[\left|\phi_{N_{h}}\right\rangle, \hat{S}_{x}\right] .
$$

In Fig. 2(b) we show $F_{Q}\left[\left|\phi_{N_{h}}\right\rangle, \hat{S}_{x}\right]$ as a function of $N_{h}$. Large values of the QFI are observed up to $N_{h} \simeq N / 2$, in accordance with the presence of macroscopic superposition states [20]. As can be seen from the Husimi distributions in Fig. 2(c), for $N_{h}<N / 2$ the $\left|\phi_{N_{h}}\right\rangle$ resemble NOON states along $\hat{S}_{x}$. This explains the Heisenberg scaling of the QFI (9).

We note that the $\hat{\mathbf{S}}$ manifold can be manipulated experimentally by radio-frequency pulses coupling the $m_{f}=0$ to the $m_{f}= \pm 1$ modes. An atomic clock using transformations in the $\hat{\mathbf{S}}$ manifold of a spin-1 BEC was demonstrated in [11], see also $[7,8,10]$ for squeezing of the $\hat{\mathbf{S}}$ spin. Our results thus 

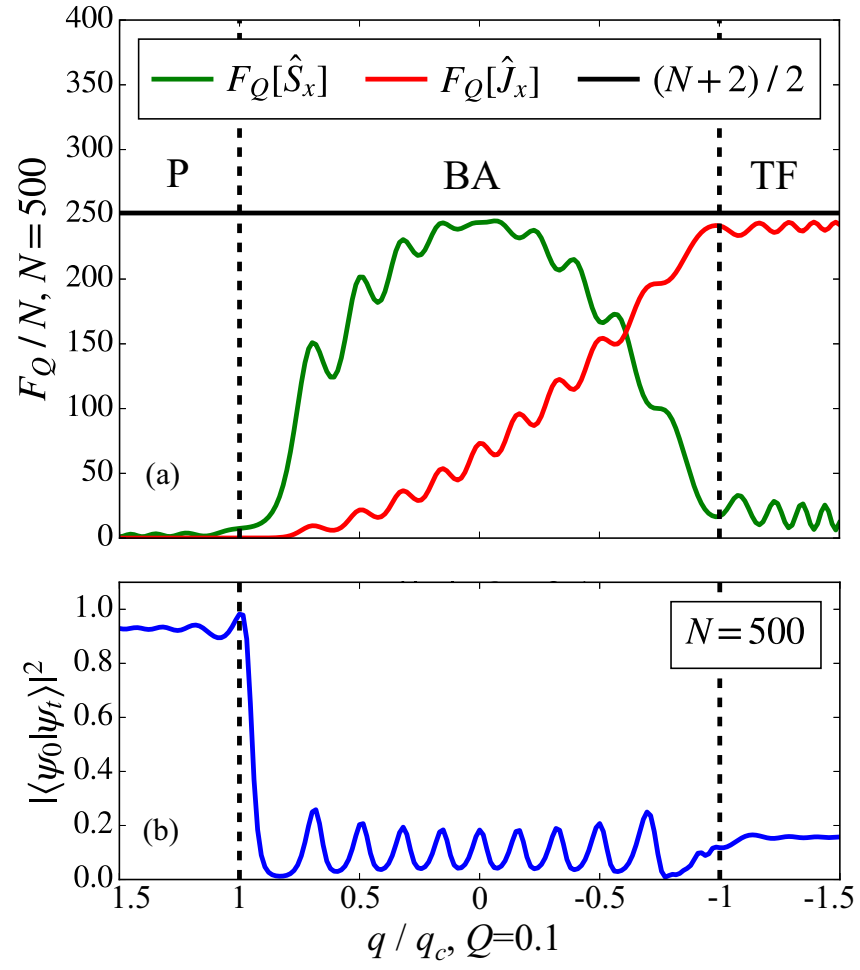

FIG. 3. Quasiadiabatic evolution of a ferromagnetic spin-1 BEC initialized in $m_{f}=0$. The quadratic Zeeman shift $q(t)$ is linearly ramped with a slope $\propto Q$. The quantum phase transitions are indicated by dashed vertical lines. (a) $F_{Q} / N$, where the interferometric transformation is generated by $\hat{S}_{x}$ (green) and $\hat{J}_{x}$ (red), which are optimal for $|\mathrm{CBA}\rangle$ and $|\mathrm{TF}\rangle$, respectively. The solid horizontal line is $F_{Q} / N=(N+2) / 2$, for comparison. (b) Overlap $\left|\left\langle\psi(t) \mid \psi_{0}(q(t))\right\rangle\right|^{2}$ of the time-evolved state $|\psi(t)\rangle$ with the ground state at $q(t)$.

reveal the possibility to attain a sensitivity close to the HL preparing the spin-1 BEC in its ground state at $q=0$. Since, when starting with the $m_{f}=0$ BEC, $q=0$ is reached after an adiabatic variation of $q$ that is half as large as the one required to arrive in the TF regime, implementing $|\mathrm{CBA}\rangle$ is less demanding in terms of BEC stability than the experiment reported in [17].

Finally, in Appendix A 3 we prove that a measurement of $\left(\hat{N}_{+}, \hat{N}_{-}\right)$is, at any $\theta$, optimal for both $|\mathrm{CBA}\rangle$ and $|\mathrm{TF}\rangle$. Optimal interferometric transformations $\hat{R}_{\text {opt }}^{(\mathrm{TF})}$ leave the TF state in the $N_{0}=0$ subspace, thus rendering $\left(\hat{N}_{+}, \hat{N}_{-}\right)$equivalent to $\hat{D}$. A similar argument, see Appendix A 3, applies to |CBA $\rangle$. Hence for both states and any phase $\theta$ the experimentally relevant measurement of $\hat{D}$ turns out to be optimal.

\section{QUASIADIABATIC STATE PREPARATION}

Next we consider an experimental sequence for the variation of $q(t)$ as the one recently discussed in [17]. We assume a BEC prepared at $q / q_{c}=1.5$ in the $\mathrm{P}$ phase where $|\psi(t=0)\rangle=$ $|k=0\rangle$. The value of the quadratic Zeeman term is varied following the ramp $q(t) / q_{c}=1.5-q_{c} Q t / 4$, where $Q>0$ characterizes the nonadiabaticity of the process. Figure 3 illustrates our observations for $N=500$ particles and $Q=$ 0.1 . We find that the QFI is hardly affected by the finite ramping speed. This is particularly striking since, as demonstrated in
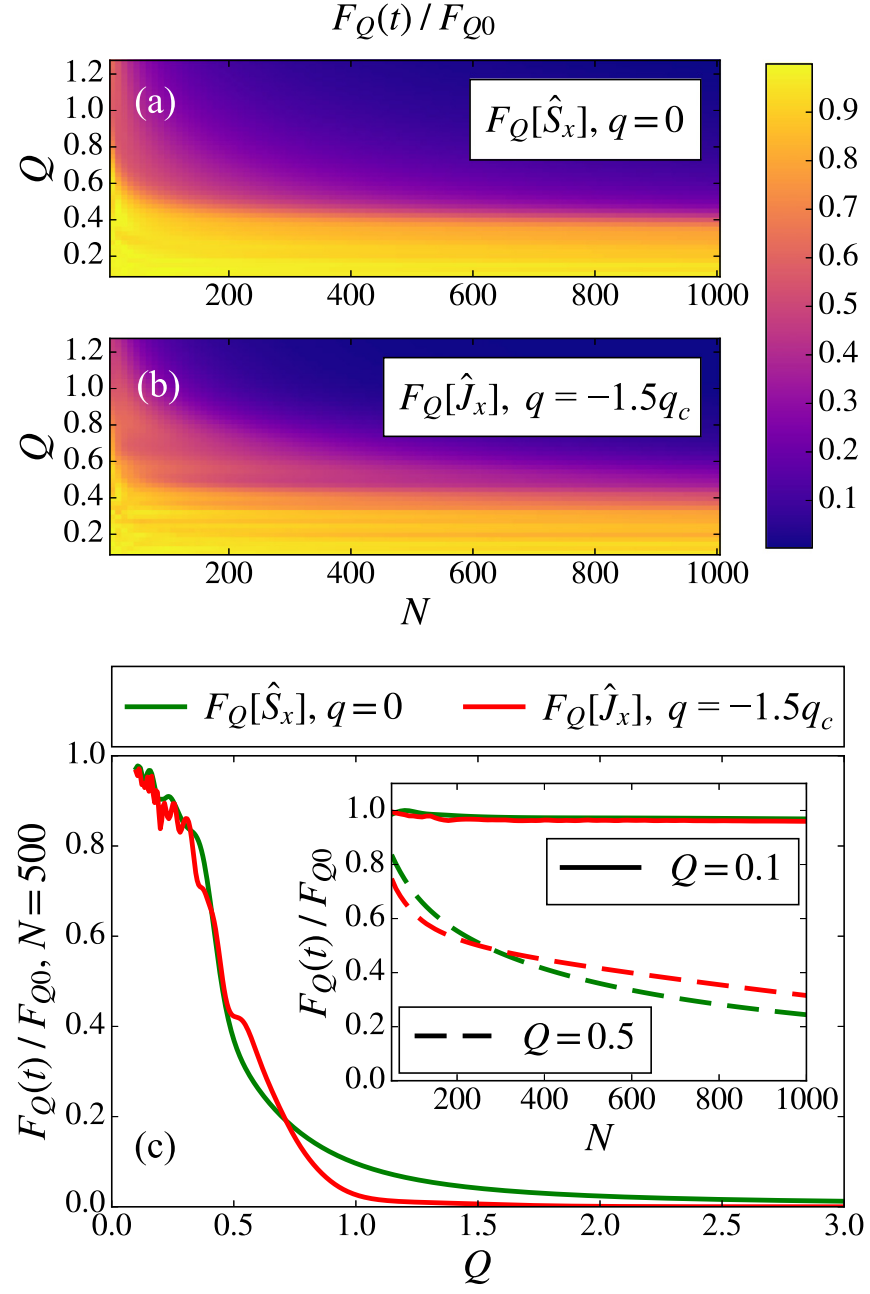

FIG. 4. (a) QFI of the states obtained via quasiadiabatic evolution up to $q=0$ and (b) $q=-1.5 q_{c}$ normalized to the QFI of the respective ground states $|\mathrm{CBA}\rangle$ and $|\mathrm{TF}\rangle$, indicated as $F_{Q 0}$. (c) Normalized QFI as a function of $Q$. The green line corresponds to $q=0$, the red line to $q=-1.5 q_{c}$. The inset shows the normalized QFI as a function of $N$. In all panels, the interferometric transformations are generated by $\hat{S}_{x}$ and $\hat{J}_{x}$ as is optimal for $|\mathrm{CBA}\rangle$ and $|\mathrm{TF}\rangle$, respectively.

Fig. 3(b), the fidelity $\left|\left\langle\psi(t) \mid \psi_{0}(q(t))\right\rangle\right|^{2}$ with the respective ground state $\left|\psi_{0}\right\rangle$ is dramatically diminished. The oscillations present in both Figs. 3(a) and 3(b) resemble the ones found in [17] for the conversion efficiency $\left\langle\hat{N}_{+}+\hat{N}_{-}\right\rangle / N$.

Note that at the critical points the energy gap between the ground and first excited state scales $\propto N^{-1 / 3}$ [13], and that a larger $Q$ means that the phase boundaries are crossed more rapidly. Hence, enlarging $N$ or $Q$ displaces $|\psi(t)\rangle$ further away from the respective ground state, creating a larger number of excitations. Figure 4 shows which fraction of the QFI for $|\mathrm{CBA}\rangle$ and $|\mathrm{TF}\rangle$ is accessible within finite time. As expected, it decreases with both $N$ and $Q$. Note that we vary $N$ at a constant but arbitrary value of $q_{c}$. In Fig. 4(c) we show slices through $F_{Q}(N, Q)$ at fixed $N$ or $Q$, respectively. The wavy distortions are due to the mentioned oscillations in the QFI, whose frequency depends both on $Q$ and (less pronounced) on $N$. We find large parts of the QFI conserved 
during non-adiabatic evolutions with $Q \lesssim 0.5$, and an overall rather small dependence on $N$. These features significantly ease experiments. Note particularly that at constant $Q$ and $\lambda$ the overall ramping time scales linearly with $1 / N$, while the factual (dimensionful) speed of the linear ramp goes even as $d q / d t \propto N^{2}$. Together with Figs. 4(a) and 4(b) this implies that enlarging the particle number at constant $\lambda$ reduces the requirements on adiabaticity and BEC stability. A pronounced dependence on whether the ramp of $q(t)$ is terminated at $q=0$ (CBA) or $q=-1.5 q_{c}$ (TF) is not discernible. This is consistent with the numerical analysis in [17] showing that the second phase transition, in contrast to the first one, has but little impact on the amount of created excitations.

\section{FINITE MEASUREMENT PRECISION}

To investigate the impact of a finite measurement resolution, we assume that the detection of both $\hat{N}_{ \pm}$is affected by Gaussian noise with variance $\sigma^{2}$, leading to an imprecise measurement of the number of particles. In this case, the actual measurement probabilities ensue from a convolution of the ideal quantum theoretical result with a Gaussian probability distribution $\Gamma_{\sqrt{2} \sigma}(x)$ of variance $2 \sigma^{2}$ and zero mean. We thus determine the classical FI from the effective probability distribution

$$
P_{\mathrm{eff}}(D \mid \theta)=\sum_{D^{\prime}=-N}^{N} \Gamma_{\sqrt{2} \sigma}\left(D-D^{\prime}\right) P\left(D^{\prime} \mid \theta\right),
$$

where $P\left(D^{\prime} \mid \theta\right)$ is the noiseless probability to find $D^{\prime}$ upon measuring $\hat{D}$ at a phase $\theta$. Figure 5 illustrates how the FI is affected by the detection uncertainty. Figure 5(a) shows for both $|\mathrm{CBA}\rangle$ and $|\mathrm{TF}\rangle$ that, while $F(\theta)$ as a whole is strongly damped, pronounced maxima at small $\theta$ remain far above the SQL, in close analogy to experiments presented in [6]. From Fig. 5(b) we infer that for worse than single particle detection $(\sigma=1 / 2)$ these peak values of the FI decrease approximately $\propto \sigma^{-2}$. Evaluating the relative standard deviation $\sigma_{\max }(N) / \sqrt{N}$ up to which the FI yet exceeds the SQL we have found that the TF state is slightly less sensitive to particle counting noise than the CBA state: $\sigma_{\max }[|\mathrm{TF}\rangle] / \sqrt{N} \approx 0.4$ while $\sigma_{\max }[|\mathrm{CBA}\rangle] / \sqrt{N} \approx$ 0.2 . Both $\sigma_{\max } / \sqrt{N}$ are easily undercut by state-of-the-art experiments [6].

\section{PARAMETRIC AMPLIFICATION}

Finally, we compare the (quasi)adiabatic state preparation with the dynamical generation of entanglement following a quench of $q$. Such a quench may render the initial $m_{f}=0$ condensate dynamically unstable. Spin-changing collisions populate $m_{f}= \pm 1$, thereby generating entanglement [26,2933].

Assuming $\left\langle\hat{N}_{ \pm}\right\rangle \ll N$, in line with experiments $[8,10-$ 12], we may approximate $N_{0} \simeq N$ and $\hat{a}_{0}^{\dagger}, \hat{a}_{0} \simeq \sqrt{N_{0}}$, which simplifies the Hamiltonian (5) to $\hat{H}=\hat{H}_{g}+$ $\hat{H}_{h} \quad$ with $\hat{H}_{g}=\alpha \hat{g}^{\dagger} \hat{g}+\beta\left(\hat{g}^{2}+\hat{g}^{\dagger 2}\right) / 2$ and $\hat{H}_{h}=\alpha \hat{h}^{\dagger} \hat{h}-$ $\beta\left(\hat{h}^{2}+\hat{h}^{\dagger 2}\right) / 2$, where $\alpha=q+\lambda(N-1 / 2)$ and $\beta=N \lambda$.

As before, the generated entanglement can be used for interferometric transformations in the symmetric and antisymmetric subspaces. The corresponding QFI of the state $|\psi(t)\rangle$ obtained by free evolution after a quench at $t=0$
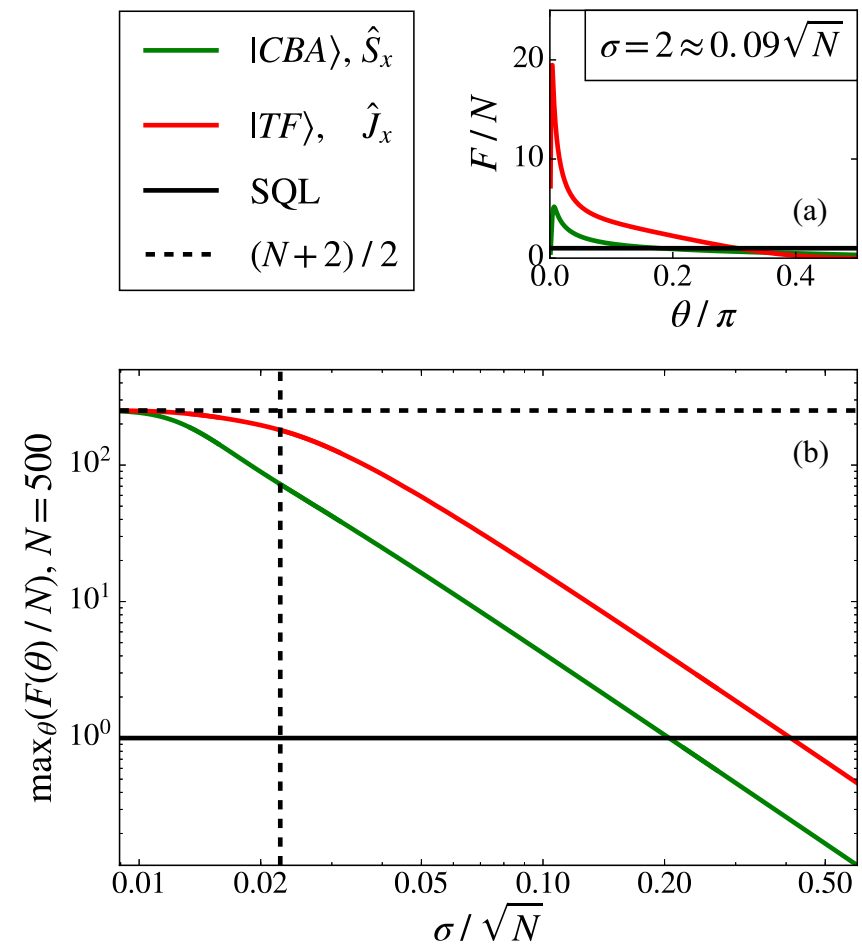

FIG. 5. FI in the presence of Gaussian measurement uncertainty with variance $\sigma^{2}$ for both $N_{ \pm}$. (a) Dependence of the FI on the interferometric phase $\theta$. (b) Peak value of the FI as a function of $\sigma$. The dashed vertical line indicates measurement noise with a spread corresponding to a single particle $(\sigma=1 / 2)$. In this Figure $N=500$.

reaches

$$
\frac{1}{N} F_{Q}\left[|\psi(t)\rangle, \hat{R}_{\mathrm{opt}}^{(g / h)}\right]=1+2\left(\left\langle\hat{N}_{ \pm}\right\rangle+\Delta \hat{N}_{ \pm}\right)
$$

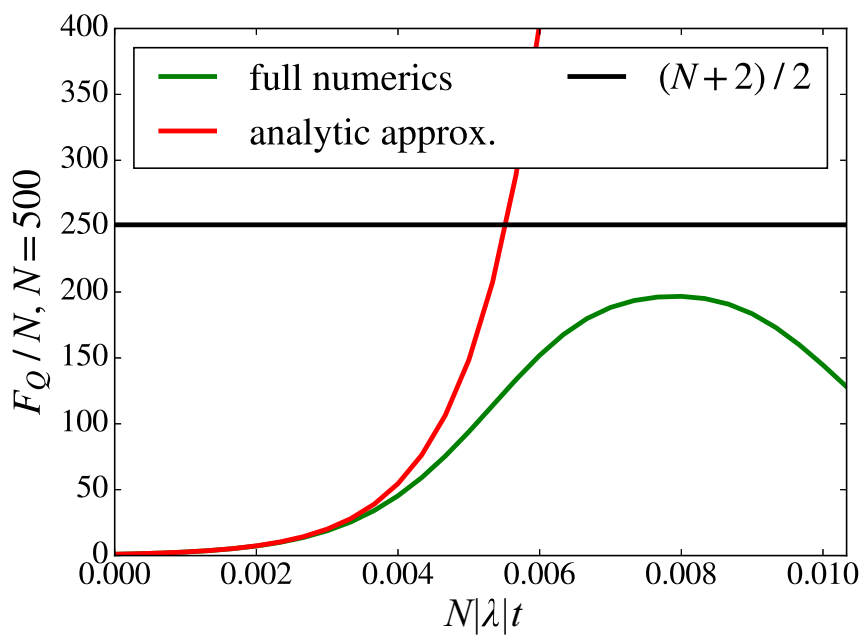

FIG. 6. QFI attainable by a quench of the quadratic Zeeman shift to its resonance value $q_{r}$ and subsequent free evolution of the $m_{f}=0$ BEC. In the experimentally relevant regime [12], the analytic (red) approximation (15) for small $t$ coincides with the full numeric simulation (green) of Hamiltonian (5). Even at times significantly exceeding current technical feasibility the QFI fails to reach the level of the TF state indicated by the threshold (black). 
with $\left(\Delta \hat{N}_{ \pm}\right)^{2}=\left\langle\hat{N}_{ \pm}\right\rangle\left(\left\langle\hat{N}_{ \pm}\right\rangle+1\right),\left\langle\hat{N}_{ \pm}\right\rangle=(N \lambda)^{2} \tau^{2} \sinh ^{2}(t / \tau)$, and $\tau=\left(\beta^{2}-\alpha^{2}\right)^{-1 / 2}$, where $\hat{R}_{\mathrm{opt}}^{(g / h)}$ is the respective generator which maximizes the QFI. This expression is valid only for short times, as long as the assumption $\left\langle\hat{N}_{ \pm}\right\rangle \ll N$ holds. The explicit form of $\hat{R}_{\mathrm{opt}}^{(g / h)}$ and a derivation of Eq. (15) are presented in Appendix B. Tuning $q$ allows to arbitrarily choose $\alpha$. At $\alpha=0$ the Hamiltonian is reduced to spin-changing collisions only. As expected, this affords the strongest growth of $\left\langle\hat{N}_{ \pm}\right\rangle$and thus of $F_{Q}$, entailing the definition of the resonance value $q_{r}=$ $-\lambda(N-1 / 2)$. Applying Eq. (15) to recent spin squeezing experiments $[8,10,11]$ provides a relative $\mathrm{QFI}, F_{Q} / N$, which ranges from 5 to 20 , thereby corresponding to a $F_{Q} / N^{2}$ of less than $2 \times 10^{-2}$ only. Recall that, in the ideal case, quasiadiabatic entanglement generation as discussed in this paper allows for $F_{Q} / N^{2} \approx 0.5$.

In the absence of technical noise it would be advantageous to extend parametric amplification protocols to longer evolution times. We therefore numerically simulate the anticipated further evolution of the QFI under the full Hamiltonian (5) at $q=q_{r}$. Figure 6 indicates that even under ideal experimental conditions parametric amplification is unable to reach the QFI attainable by the quasiadiabatic approach. In the best scenario, dynamical spin-changing collision creates entangled states with a QFI $F_{Q} / N^{2} \approx 0.257$ [14].

\section{CONCLUSION}

We study the generation of entanglement useful for quantum enhanced interferometry with ferromagnetic spin-1 BECs, focusing on the experimentally relevant case of quasiadiabatic driving through quantum phase transitions. We show that, starting out from a BEC in $m_{f}=0$ and a quadratic Zeeman shift of $q>q_{c}$, at $q=0$ and thus halfway to the TF state another highly entangled state, $|\mathrm{CBA}\rangle$, of equal interferometric value (approximately equal QFI) emerges. This allows us to propose an alternative interferometric scheme admitting Heisenberg scaling.

For both $|\mathrm{TF}\rangle$ and $|\mathrm{CBA}\rangle$, optimal values of the QFI are obtained with interferometric transformations corresponding to common radio-frequency and microwave coupling techniques. The optimal measurement procedure is based on the well established counting of particles in the $m_{f}= \pm 1$ modes. According to our findings surpassing the standard quantum limit is expected to remain feasible under realistic conditions, when the quasiadiabatic transition is performed at finite speed, and measurement uncertainty is present. While the TF state is less sensitive to imperfections of atom counting, the CBA state has the advantage of being quasiadiabatically reachable within half the time. Both regimes favorably compare to squeezing through parametric amplification, thus constituting a promising source of interferometrically useful entanglement.

Note added in proof: Recently, a quasiadiabatic preparation of the CBA state and its successful application to atom interferometry beyond the SQL have been reported in [34].

\section{ACKNOWLEDGMENTS}

We acknowledge support by the SFB 1227 "DQ-mat" Projects No. A02 and No. B01, of the German Research
Foundation (DFG). M. G. thanks the Alexander von Humboldt foundation for support.

\section{APPENDIX A: ADIABATIC PHASE TRANSITION}

\section{Gell-Mann covariance matrix}

The collective Gell-Mann operators read

$$
\begin{aligned}
& \hat{G}_{1}=\frac{\hat{a}_{-1}^{\dagger} \hat{a}_{0}+\hat{a}_{0}^{\dagger} \hat{a}_{-1}}{2}, \quad \hat{G}_{2}=\frac{\hat{a}_{-1}^{\dagger} \hat{a}_{0}-\hat{a}_{0}^{\dagger} \hat{a}_{-1}}{2 i}, \\
& \hat{G}_{3}=\frac{\hat{a}_{-1}^{\dagger} \hat{a}_{-1}-\hat{a}_{0}^{\dagger} \hat{a}_{0}}{2}, \quad \hat{G}_{4}=\frac{\hat{a}_{1}^{\dagger} \hat{a}_{-1}+\hat{a}_{-1}^{\dagger} \hat{a}_{1}}{2}, \\
& \hat{G}_{5}=\frac{\hat{a}_{-1}^{\dagger} \hat{a}_{1}-\hat{a}_{1}^{\dagger} \hat{a}_{-1}}{2 i}, \quad \hat{G}_{6}=\frac{\hat{a}_{0}^{\dagger} \hat{a}_{1}+\hat{a}_{1}^{\dagger} \hat{a}_{0}}{2}, \\
& \hat{G}_{7}=\frac{\hat{a}_{0}^{\dagger} \hat{a}_{1}-\hat{a}_{1}^{\dagger} \hat{a}_{0}}{2 i}, \quad \hat{G}_{8}=\frac{\hat{a}_{-1}^{\dagger} \hat{a}_{-1}+\hat{a}_{0}^{\dagger} \hat{a}_{0}-2 \hat{a}_{1}^{\dagger} \hat{a}_{1}}{2 \sqrt{3}} .
\end{aligned}
$$

Consider an arbitrary (normalized) state $|\psi\rangle=\sum_{k} c_{k}|k\rangle$ with $\hat{D}|\psi\rangle=0$. The covariance matrix of $\hat{\mathbf{G}}$ is block diagonal

$$
\boldsymbol{\Gamma}_{\hat{\mathbf{G}}}=\boldsymbol{\Gamma}_{\left(\hat{G}_{1}, \hat{G}_{2}, \hat{G}_{6}, \hat{G}_{7}\right)} \oplus \boldsymbol{\Gamma}_{\left(\hat{G}_{3}, \hat{G}_{8}\right)} \oplus\left(\Delta \hat{G}_{4}\right)^{2} \oplus\left(\Delta \hat{G}_{5}\right)^{2}
$$

with $\left(\Delta \hat{G}_{i}\right)^{2}$ the variance of $\hat{G}_{i}$. The twofold degenerate eigenvalues of $\boldsymbol{\Gamma}_{\left(\hat{G}_{1}, \hat{G}_{2}, \hat{G}_{6}, \hat{G}_{7}\right)}$ are

$$
\lambda_{ \pm}=A \pm|B|
$$

with

$$
\begin{aligned}
& A=\frac{1}{4}\left(N+\sum_{k=0}^{\lfloor N / 2\rfloor}\left|c_{k}\right|^{2} k(2 N-4 k-1)\right), \\
& B=\frac{1}{2} \sum_{k=0}^{\lfloor N / 2-1\rfloor} c_{k}^{*} c_{k+1}(k+1) \sqrt{(N-2 k)(N-2 k-1)}
\end{aligned}
$$

and corresponding eigenvectors

$$
\begin{aligned}
& \mathbf{u}_{+}^{(1)}=\frac{1}{\sqrt{2}|B|}(\operatorname{Im} B, \operatorname{Re} B, 0,|B|), \\
& \mathbf{u}_{+}^{(2)}=\frac{1}{\sqrt{2}|B|}(\operatorname{Re} B,-\operatorname{Im} B,|B|, 0), \\
& \mathbf{u}_{-}^{(1)}=\frac{1}{\sqrt{2}|B|}(-\operatorname{Im} B,-\operatorname{Re} B, 0,|B|), \\
& \mathbf{u}_{-}^{(2)}=\frac{1}{\sqrt{2}|B|}(-\operatorname{Re} B, \operatorname{Im} B,|B|, 0),
\end{aligned}
$$

where Re and Im denote the real and imaginary parts, respectively. Note that if $c_{k} \in \mathbb{R}$ for all $k$ we obtain $\operatorname{Im} B=0$ and hence $\boldsymbol{\Gamma}_{\left(\hat{G}_{1}, \hat{G}_{2}, \hat{G}_{6}, \hat{G}_{7}\right)}=\boldsymbol{\Gamma}_{\left(\hat{G}_{1}, \hat{G}_{6}\right)} \oplus \boldsymbol{\Gamma}_{\left(\hat{G}_{2}, \hat{G}_{7}\right)}$. The eigenvalues of $\Gamma_{\left(\hat{G}_{3}, \hat{G}_{8}\right)}$ are

$$
\lambda_{0}=0, \quad \lambda_{1}=3\left(\sum_{k=0}^{\lfloor N / 2\rfloor}\left|c_{k}\right|^{2} k^{2}-\left(\sum_{k=0}^{\lfloor N / 2\rfloor}\left|c_{k}\right|^{2} k\right)^{2}\right) .
$$

The corresponding eigenvectors read

$$
\mathbf{u}_{0}=\frac{1}{2}(1, \sqrt{3}), \quad \mathbf{u}_{1}=\frac{1}{2}(\sqrt{3},-1) .
$$


Finally,

$$
\left(\Delta \hat{G}_{4}\right)^{2}=\left(\Delta \hat{G}_{5}\right)^{2}=\frac{1}{2} \sum_{k=0}^{\lfloor N / 2\rfloor}\left|c_{k}\right|^{2} k(k+1) .
$$

Numerically evaluating $\boldsymbol{\Gamma}_{\hat{\mathbf{G}}}$ in the ground state $\left|\psi_{0}(q)\right\rangle$ of the Hamiltonian (5) we find that only the (two-fold degenerate) eigenvalues $\lambda_{+}$and $\left(\Delta \hat{G}_{4}\right)^{2}=\left(\Delta \hat{G}_{5}\right)^{2}$, depicted in Figs. 1 and 3(a), significantly differ from zero for large $N$. They correspond to the eigenoperators $\hat{S}_{x}=\left(\hat{G}_{1}+\hat{G}_{6}\right) / \sqrt{2}, \hat{A}_{y}=$ $\left(\hat{G}_{2}+\hat{G}_{7}\right) / \sqrt{2}$, which become optimal in the BA phase, and $\hat{J}_{x}=\hat{G}_{4}, \hat{J}_{y}=-\hat{G}_{5}$, prevalent in the TF phase, respectively.

\section{Properties of the CBA state}

In this section, we provide some analytical results on the CBA state, i.e., the ground state at $q=0$.

\section{a. Coefficients in the Fock basis}

Let us introduce the collective pseudospin-1 operator $\hat{\mathbf{L}}$ composed of

$$
\begin{aligned}
& \hat{L}_{x}=\frac{1}{\sqrt{2}}\left(\hat{a}_{0}^{\dagger} \hat{a}_{1}+\hat{a}_{0}^{\dagger} \hat{a}_{-1}+\hat{a}_{1}^{\dagger} \hat{a}_{0}+\hat{a}_{-1}^{\dagger} \hat{a}_{0}\right)=2 \hat{S}_{x}, \\
& \hat{L}_{y}=\frac{1}{i \sqrt{2}}\left(\hat{a}_{0}^{\dagger} \hat{a}_{1}-\hat{a}_{0}^{\dagger} \hat{a}_{-1}-\hat{a}_{1}^{\dagger} \hat{a}_{0}+\hat{a}_{-1}^{\dagger} \hat{a}_{0}\right)=2 \hat{A}_{y}, \\
& \hat{L}_{z}=\hat{a}_{-1}^{\dagger} \hat{a}_{-1}-\hat{a}_{1}^{\dagger} \hat{a}_{1}=-2 \hat{J}_{z} .
\end{aligned}
$$

This allows us to express the ground state of the Hamiltonian (5) in the subspace of $D=0$ at $q=0$ as [25]

$$
\begin{aligned}
|\mathrm{CBA}\rangle & =\frac{1}{\sqrt{(2 N) !}}\left(\hat{L}_{+}\right)^{N}\left|N_{-}=0, N_{0}=0, N_{+}=N\right\rangle, \\
\hat{L}_{+} & =\hat{L}_{x}+i \hat{L}_{y}=\sqrt{2}\left(\hat{a}_{-1}^{\dagger} \hat{a}_{0}+\hat{a}_{0}^{\dagger} \hat{a}_{1}\right) .
\end{aligned}
$$

We expand the operator $\hat{L}_{+}^{N}$, leading to

$$
\begin{aligned}
|\mathrm{CBA}\rangle & =\sqrt{\frac{2^{N}}{(2 N) !}} \sum_{k=0}^{\lfloor N / 2\rfloor} \mathfrak{C}_{k}\left[\hat{a}_{0}, \hat{a}_{0}^{\dagger}\right] \hat{a}_{-1}^{\dagger k} \hat{a}_{1}^{N-k}|0,0, N\rangle \\
& =\sqrt{\frac{2^{N} N !}{(2 N) !}} \sum_{k=0}^{\lfloor N / 2\rfloor} \mathfrak{C}_{k}\left[\hat{a}_{0}, \hat{a}_{0}^{\dagger}\right]|k, 0, k\rangle,
\end{aligned}
$$

where $\mathfrak{C}_{k}\left[\hat{a}, \hat{a}^{\dagger}\right]$ denotes the sum over all possible products composed of $k$ operators $\hat{a}$ and $N-k$ operators $\hat{a}^{\dagger}$ in arbitrary order, and we have used that $\hat{a}_{-1}^{\dagger}$ and $\hat{a}_{1}$ commute with each other as well as with $\hat{a}_{0}$ and $\hat{a}_{0}^{\dagger}$. Each term in $\mathfrak{C}_{k}\left[\hat{a}_{0}, \hat{a}_{0}^{\dagger}\right]$ leads to a total creation of $N-2 k$ particles in mode $\hat{a}_{0}$. Since we apply $\hat{L}_{+}^{N}$ to a state containing zero particles in the central mode, only values of $k \leqslant N / 2$ contribute to Eq. (A11). An explicit evaluation can be performed by means of the following lemma.

Lemma 1: For an arbitrary bosonic mode with creation operator $\hat{a}^{\dagger}$ and vacuum $|0\rangle$

$$
\mathfrak{C}_{k}\left[\hat{a}, \hat{a}^{\dagger}\right]|0\rangle=\frac{N !}{2^{k} k !(N-2 k) !}\left(\hat{a}^{\dagger}\right)^{N-2 k}|0\rangle \quad \forall k \leqslant N / 2 .
$$

Proof. Since each term in the sum described by $\mathfrak{C}_{k}\left[\hat{a}, \hat{a}^{\dagger}\right]$ describes the creation of $N-2 k$ particles, we can write

$$
\mathfrak{C}_{k}\left[\hat{a}, \hat{a}^{\dagger}\right]|0\rangle=X(k)\left(\hat{a}^{\dagger}\right)^{N-2 k}|0\rangle,
$$

which reduces the problem to the identification of the combinatorial factor $X(k)$. We use Wick's theorem [35], $\hat{a}|0\rangle=0$, and the fundamental Wick contractions

$$
\begin{array}{r}
\hat{a} \hat{a}=\hat{a}^{\dagger} \hat{a}^{\dagger}=\underbrace{\hat{a}^{\dagger} \hat{a}}=0, \\
\underline{\underline{a}}^{\hat{a}^{\dagger}}=1
\end{array}
$$

where $\hat{A} \hat{B} \equiv \hat{A} \hat{B}-: \hat{A} \hat{B}$ : and the double dots denote normal ordering. The contribution of each permutation to $X(k)$ is the number of variants it admits for enclosing all $k$ annihilation operators into $\hat{a} \hat{a}^{\dagger}$ contractions. Taking into consideration all permutations of $\hat{a}, \hat{a}^{\dagger}$ reveals that $X(k)$ is the number of possibilities to tag $k$ unsorted disjoint tuples in a set of $N$ elements. There are $\left(\begin{array}{c}N \\ N-2 k\end{array}\right)$ different choices for the positions of the $\hat{a}^{\dagger}$ which are not going to be contracted. Thus, we merely have to count the number of possibilities to pair $2 k$ objects. First arbitrarily arranging them and then compensating for the ordering of and within pairs we arrive at $(2 k) ! /\left(2^{k} k !\right)$. This completes the proof, since

$$
X(k)=\left(\begin{array}{c}
N \\
N-2 k
\end{array}\right) \frac{(2 k) !}{2^{k} k !}=\frac{N !}{2^{k} k !(N-2 k) !} .
$$

Applying Lemma 1 to Eq. (A11) gives

$$
|\mathrm{CBA}\rangle=\sqrt{\frac{2^{N} N ! 3}{(2 N) !}} \sum_{k=0}^{\lfloor N / 2\rfloor} \frac{1}{2^{k} k ! \sqrt{(N-2 k) !}}|k, N-2 k, k\rangle
$$

as reported in Eq. (10).

\section{b. Quantum Fisher information}

As discussed in the main text, see Fig. 1, and in Appendix A 1 , the QFI of $|\mathrm{CBA}\rangle$ is maximized by any $\hat{R}_{\text {opt }} \in$ $\operatorname{span}\left\{\hat{S}_{x}, \hat{A}_{y}\right\}$. We consider without loss of generality $\hat{R}_{\mathrm{opt}}=$ $\hat{S}_{x}$. Then

$$
\begin{aligned}
F_{Q}\left[|\mathrm{CBA}\rangle, \hat{R}_{\mathrm{opt}}\right]= & 4\left(\Delta \hat{S}_{x}\right)^{2} \\
= & \sum_{k=0}^{\lfloor(N-1) / 2\rfloor}(k+1) \mid \sqrt{N-2 k} c_{k} \\
& +\left.\sqrt{N-2 k-1} c_{k+1}\right|^{2},
\end{aligned}
$$

where

$$
c_{k}=\sqrt{\frac{2^{N} N !^{3}}{(2 N) !}} \frac{1}{2^{k} k ! \sqrt{(N-2 k) !}}
$$

are the Fock-state coefficients of $|\mathrm{CBA}\rangle$ from Eq. (A16), and $c_{k>N / 2} \equiv 0$. Thus

$$
\begin{aligned}
& \sqrt{N-2 k} c_{k}+\sqrt{N-2 k-1} c_{k+1} \\
& =\sqrt{\frac{2^{N} N !^{3}}{(2 N) !}} \frac{N+1}{2^{k+1}(k+1) ! \sqrt{(N-2 k-1) !}},
\end{aligned}
$$


which, after some rearrangements, leads to

$$
\begin{aligned}
F_{Q}\left[|\mathrm{CBA}\rangle, \hat{R}_{\mathrm{opt}}\right]= & \frac{(N+1) !^{2}}{2(2 N) !} \\
& \times \sum_{k=0}^{\lfloor(N-1) / 2\rfloor}\left(\begin{array}{l}
N \\
k
\end{array}\right)\left(\begin{array}{l}
N-k \\
k+1
\end{array}\right) 2^{N-2 k-1} .
\end{aligned}
$$

The sum ensues from the following lemma.

Lemma 2:

$$
\sum_{k=0}^{\lfloor n / 2\rfloor}\left(\begin{array}{c}
m \\
k
\end{array}\right)\left(\begin{array}{c}
m-k \\
n-2 k
\end{array}\right) 2^{n-2 k}=\left(\begin{array}{c}
2 m \\
n
\end{array}\right) \forall n \leqslant 2 m .
$$

Proof. Consider $2 m$ sites grouped into $m$ pairs. For each $k$ the left-hand side of Eq. (A21) is the number of possibilities to distribute $2 k+(n-2 k)=n$ indiscernible objects on these $2 m$ sites in such a way that exactly $k$ pairs are completed. The number of obtained pairs can assume values between $\max \{0, n-m\}$ and $\lfloor n / 2\rfloor$. Note that $k<n-m$ do not contribute to Eq. (A21). Thus, summing over all $0 \leqslant k \leqslant n / 2$ amounts to counting the variants of distributing $n$ identical elements on $2 m$ sites, which gives $\left(\begin{array}{c}2 m \\ n\end{array}\right)$.

Choosing $n=N-1$ and $m=N$ we obtain

$$
F_{Q}\left[|\mathrm{CBA}\rangle, \hat{R}_{\mathrm{opt}}\right]=N(N+1) / 2 \text {. }
$$

\section{c. Mean particle number}

We further determine the exact mean particle numbers in the $\hat{a}_{ \pm 1}$ modes, using

$$
\left\langle\hat{N}_{ \pm}\right\rangle=\sum_{k} k\left|c_{k}\right|^{2},
$$

with the coefficients of the CBA state defined in Eq. (A18). Applying Lemma 2 with $n=N$ and $m=N-1$, we find

$$
\begin{aligned}
\left\langle\hat{N}_{ \pm}\right\rangle & =\frac{N}{4} \times \frac{2 N-2}{2 N-1} \\
& =\frac{N}{4}\left[1-\frac{1}{2 N}+\mathcal{O}\left(\frac{1}{N^{2}}\right)\right] .
\end{aligned}
$$

For $N \gg 1$, we recover the mean field expression $\left\langle\hat{N}_{ \pm}\right\rangle=$ $N / 4$.

\section{Optimal measurements}

We consider the two interferometrically relevant states $|\psi\rangle \in\{|\mathrm{CBA}\rangle,|\mathrm{TF}\rangle\}$ along with the respective optimal generators of the interferometric transformation

$$
\begin{aligned}
\hat{R}_{\mathrm{opt}}^{(\mathrm{CBA})}(\phi)= & \frac{1}{2 \sqrt{2}}\left(e^{-i \phi} \hat{a}_{0}^{\dagger} \hat{a}_{1}+e^{i \phi} \hat{a}_{0}^{\dagger} \hat{a}_{-1}\right. \\
& \left.+e^{i \phi} \hat{a}_{1}^{\dagger} \hat{a}_{0}+e^{-i \phi} \hat{a}_{-1}^{\dagger} \hat{a}_{0}\right), \\
\hat{R}_{\mathrm{opt}}^{(\mathrm{TF})}(\phi)= & \frac{1}{2}\left(e^{-i \phi} \hat{a}_{1}^{\dagger} \hat{a}_{-1}+e^{i \phi} \hat{a}_{-1}^{\dagger} \hat{a}_{1}\right)
\end{aligned}
$$

providing $|\psi(\theta)\rangle=\mathrm{e}^{-i \theta \hat{R}_{\mathrm{opt}}}|\psi\rangle$. Let us first focus on $\phi=0$, where $\hat{R}_{\mathrm{opt}}^{(\mathrm{CBA})}(0)=\hat{S}_{x}$ and $\hat{R}_{\mathrm{opt}}^{(\mathrm{TF})}(0)=\hat{J}_{x}$, and show that a measurement of $\left(\hat{N}_{+}, \hat{N}_{-}\right)$is optimal at any $\theta$.
The projections $\hat{P}_{N_{+}, N_{-}} \equiv\left|N_{-}, N_{0}, N_{+}\right\rangle\left\langle N_{-}, N_{0}, N_{+}\right|$ with $N_{0}=N-N_{+}-N_{-}$onto the eigenstates of $\left(\hat{N}_{+}, \hat{N}_{-}\right)$ are one-dimensional. In addition, $\langle\psi \mid \mathrm{d} \psi\rangle=0$ and hence $\langle\psi(\theta) \mid \mathrm{d} \psi(\theta)\rangle=0$ for all $\theta$. Therefore

$$
\operatorname{Re}\left\langle\psi(\theta)\left|\hat{P}_{N_{+}, N_{-}} \hat{R}_{\text {opt }}\right| \psi(\theta)\right\rangle=0 \forall N_{+}, N_{-}, \theta
$$

provides both the necessary and sufficient condition for optimality [22]. We observe that $\hat{R}_{\text {opt }}^{n}|\psi\rangle, n \in \mathbb{N}_{0}$ has only real coefficients in the Fock basis $\left\{\left|N_{-}, N_{0}, N_{+}\right\rangle\right\}$. Furthermore,

$$
\begin{gathered}
\sum_{D=2 n} \hat{P}_{D} \hat{S}_{x}^{2 m+1}|\mathrm{CBA}\rangle=0, \\
\sum_{D=2 n+1} \hat{P}_{D} \hat{S}_{x}^{2 m}|\mathrm{CBA}\rangle=0
\end{gathered}
$$

and

$$
\begin{aligned}
\sum_{N_{ \pm}=2 n} \hat{P}_{N_{ \pm}} \hat{J}_{x}^{2 m+1}|\mathrm{TF}\rangle & =0, \\
\sum_{N_{ \pm}=2 n+1} \hat{P}_{N_{ \pm}} \hat{J}_{x}^{2 m}|\mathrm{TF}\rangle & =0
\end{aligned}
$$

for all $n, m \in \mathbb{N}_{0}$. This entails

$$
\begin{gathered}
\left\langle\psi\left|\hat{R}_{\mathrm{opt}}^{2 n} \hat{P}_{N_{+}, N_{-}} \hat{R}_{\mathrm{opt}}^{2 m+1}\right| \psi\right\rangle=0, \\
\left\langle\psi\left|\hat{R}_{\mathrm{opt}}^{2 n+1} \hat{P}_{N_{+}, N_{-}} \hat{R}_{\mathrm{opt}}^{2 m}\right| \psi\right\rangle=0 .
\end{gathered}
$$

Thus

$$
\begin{aligned}
& \left\langle\psi(\theta)\left|\hat{P}_{N_{+}, N_{-}} \hat{R}_{\mathrm{opt}}\right| \psi(\theta)\right\rangle \\
& =i \sum_{j, l=0}^{\infty} \frac{(-1)^{j+l}}{(2 j) !(2 l) !} \theta^{2(j+l)+1} \\
& \quad \times\left(\frac{1}{2 j+1}\left\langle\psi\left|\hat{R}_{\mathrm{opt}}^{2 j+1} \hat{P}_{N_{+}, N_{-}} \hat{R}_{\mathrm{opt}}^{2 l+1}\right| \psi\right\rangle\right. \\
& \left.\quad-\frac{1}{2 l+1}\left\langle\psi\left|\hat{R}_{\mathrm{opt}}^{2 j} \hat{P}_{N_{+}, N_{-}} \hat{R}_{\mathrm{opt}}^{2 l+2}\right| \psi\right\rangle\right) \in i \mathbb{R}
\end{aligned}
$$

which implies Eq. (A26).

Next, we consider a measurement of $\hat{D}$, still for $\phi=0$. Due to $\hat{P}_{D}=\sum_{N_{+}-N_{-}=D} \hat{P}_{N_{+}, N_{-}}$, Eq. (A26) holds also when $\hat{P}_{N_{+}, N_{-}}$is substituted by $\hat{P}_{D}$. Since the $\hat{P}_{D}$ are no longer one-dimensional, this is not sufficient for optimality [22]. However, we are able to show that for both $|\mathrm{CBA}\rangle$ and $|\mathrm{TF}\rangle$ the Hilbert space $\mathcal{H}=\operatorname{span}\left\{\left|N_{+}, N_{0}, N_{-}\right\rangle\right\}$can be restricted to $\mathcal{H}^{\prime}$ such that $\left\{\hat{R}_{\text {opt }}^{n}|\psi\rangle, n \in \mathbb{N}_{0}\right\} \in \mathcal{H}^{\prime}$ while the dimensionality of $\hat{P}_{D} \mathcal{H}^{\prime}$ for any $D$ is one. Let us start with the TF state. Since $\left[\hat{J}_{x}, \hat{N}_{0}\right]=0$ and $\hat{N}_{0}|\mathrm{TF}\rangle=0$, we can choose $\mathcal{H}^{\prime}=\hat{P}_{N_{0}=0} \mathcal{H}$. Regarding $|\mathrm{CBA}\rangle$, recall that $\hat{S}_{x}=\hat{L}_{x} / 2$. Hence $\left[\hat{S}_{x}, \hat{\mathbf{L}}^{2}\right]=0$. Then $\hat{\mathbf{L}}^{2}|\mathrm{CBA}\rangle=N(N+1)|\mathrm{CBA}\rangle[25]$ suggests to set $\mathcal{H}^{\prime}=$ $\hat{P}_{\hat{\mathbf{L}}^{2}=N(N+1)} \mathcal{H}$. $\hat{L}_{z}$ has a nondegenerate spectrum in $\mathcal{H}^{\prime}$. Thus $\hat{L}_{z}=-\hat{D}$ establishes the one-dimensionality of all $\hat{P}_{D} \mathcal{H}^{\prime}$.

To proceed to arbitrary $\phi$ we note that

$$
\begin{aligned}
\hat{R}_{\text {opt }}^{(\mathrm{CBA})}(\phi) & =\mathrm{e}^{2 i \phi \hat{J}_{z}} \hat{S}_{x} \mathrm{e}^{-2 i \phi \hat{J}_{z}}, \\
\hat{R}_{\text {opt }}^{(\mathrm{TF})}(\phi) & =\mathrm{e}^{i \phi \hat{J}_{z}} \hat{J}_{x} \mathrm{e}^{-i \phi \hat{J}_{z}}
\end{aligned}
$$

and recall that the classical FI (2) depends only on $P(\mu \mid \theta)=$ $\left\langle\psi(\theta)\left|\hat{P}_{\mu}\right| \psi(\theta)\right\rangle$ with $\mu$ the possible measurement outcomes. 
Because $e^{-i \phi \hat{J}_{z}} \hat{P}_{N_{+}, N_{-}} e^{i \phi \hat{J}_{z}}=\hat{P}_{N_{+}, N_{-}}$and, thanks to $\hat{D}|\psi\rangle=$ $0, e^{-i \phi \hat{J}_{z}}|\psi\rangle=|\psi\rangle$, our results hold for any $\phi$.

\section{APPENDIX B: PARAMETRIC AMPLIFICATION}

We start by introducing the generators of $\mathrm{SU}(1,1)$

$$
\hat{L}_{0}^{(a)}=\frac{1}{4}\left(2 \hat{a}^{\dagger} \hat{a}+1\right), \hat{L}_{+}^{(a)}=\frac{1}{2} \hat{a}^{\dagger 2}, \hat{L}_{-}^{(a)}=\frac{1}{2} \hat{a}^{2},
$$

where $\hat{a}^{\dagger}$ denotes some bosonic creation operator. Under the approximations $N_{0} \simeq N$ and $\hat{a}_{0}^{\dagger}, \hat{a}_{0} \simeq \sqrt{N_{0}}$ the Hamiltonian (5) becomes, discarding c-numbers, $\hat{H}=\hat{H}_{g}+\hat{H}_{h}$ with

$$
\hat{H}_{g / h}=2 \alpha \hat{L}_{0}^{(g / h)}+\epsilon_{g / h} \beta\left(\hat{L}_{-}^{(g / h)}+\hat{L}_{+}^{(g / h)}\right),
$$

$\epsilon_{g / h}= \pm 1$, and $\alpha=q+\lambda(N-1 / 2), \beta=N \lambda$. Let us denote the eigenstates of $\hat{N}_{g} \equiv \hat{g}^{\dagger} \hat{g}$ and $\hat{N}_{h} \equiv \hat{h}^{\dagger} \hat{h}$ by $|n\rangle_{g / h}$. The initial state $\left(m_{f}=0\right.$ condensate $) \quad|\psi(0)\rangle=|0\rangle_{g} \otimes$ $|0\rangle_{h}$ evolves as $|\psi(t)\rangle=|\psi(t)\rangle_{g} \otimes|\psi(t)\rangle_{h}$ with $|\psi(t)\rangle_{g / h} \equiv$ $\exp \left(-i \hat{H}_{g / h} t\right)|0\rangle_{g / h}$. An explicit form of $|\psi(t)\rangle_{g / h}$ is obtained from the disentanglement theorem for $\mathrm{SU}(1,1)[36]$ : for $t, r \in$ $\mathbb{R}, z \in \mathbb{C}$

$$
\begin{aligned}
& \exp \left[\left(z \hat{L}_{+}-z^{*} \hat{L}_{-}+2 i r \hat{L}_{0}\right) t\right] \\
& \quad=\exp \left(p_{+} \hat{L}_{+}\right) \exp \left(p_{0} \hat{L}_{0}\right) \exp \left(p_{-} \hat{L}_{-}\right)
\end{aligned}
$$

with $\quad p_{+}=z \tau \sinh (t / \tau) / C, \quad p_{0}=-2 \ln C, \quad p_{-}=$ $-z^{*} \tau \sinh (t / \tau) / C, \quad C=\cosh (t / \tau)-i r \tau \sinh (t / \tau), \quad$ and $\tau=\left(|z|^{2}-r^{2}\right)^{-1 / 2}$. The result for $|z|^{2}=r^{2}$ is obtained by taking the corresponding limit [36]. This entails, see also [5],

$$
|\psi(t)\rangle_{g, h}=\frac{1}{\sqrt{|C|}} \sum_{n=0}^{\infty} \epsilon_{g, h}^{n} \sqrt{\left(\begin{array}{c}
2 n \\
n
\end{array}\right)}\left(\frac{c}{2}\right)^{n}|2 n\rangle_{g, h}
$$

with $\quad c=-i \beta \tau \sinh (t / \tau) / C, \quad C=\cosh (t / \tau)+$ $i \alpha \tau \sinh (t / \tau)$, and $\tau=\left(\beta^{2}-\alpha^{2}\right)^{-1 / 2}$.

The evaluation of $\left\langle\hat{N}_{g}\right\rangle, \Delta \hat{N}_{g}$, and the QFI relies on the generating function

$$
f(z)=\sum_{n=0}^{\infty}\left(\begin{array}{c}
2 n \\
n
\end{array}\right)\left(\frac{|c|}{2}\right)^{2 n} e^{n z}=\frac{1}{\sqrt{1-|c|^{2} e^{z}}}
$$

for $|c|^{2} e^{z}<1$. We first derive $\left\langle\hat{N}_{g}\right\rangle=\beta^{2} \tau^{2} \sinh ^{2}(t / \tau)$ and $\left(\Delta \hat{N}_{g}\right)^{2}=2\left\langle\hat{N}_{g}\right\rangle\left(\left\langle\hat{N}_{g}\right\rangle+1\right)$. Applying $N_{0} \simeq N$ and $\hat{a}_{0}^{\dagger}, \hat{a}_{0} \simeq$ $\sqrt{N_{0}}$ also to $\hat{\mathbf{S}}$ gives

$$
\hat{S}_{x} \simeq \frac{\sqrt{N}}{2}\left(\hat{g}+\hat{g}^{\dagger}\right), \quad \hat{S}_{y} \simeq \frac{\sqrt{N}}{2 i}\left(\hat{g}-\hat{g}^{\dagger}\right), \quad \hat{S}_{z} \simeq \frac{1}{2}\left(N-\hat{g}^{\dagger} \hat{g}\right) .
$$

The corresponding covariance matrix $\boldsymbol{\Gamma}_{\hat{\mathbf{S}}}$ is block diagonal. The eigenvalues of its $(x, y)$ block evaluate to

$$
\lambda_{ \pm}^{(x y)}=\frac{N}{4}\left(1+2\left\langle\hat{N}_{g}\right\rangle \pm \sqrt{2} \Delta \hat{N}_{g}\right)
$$

while the variance of $\hat{S}_{z}$ is $\lambda^{(z)}=\left(\Delta \hat{N}_{g}\right)^{2} / 4$. Thus, for $N \gg$ $\left\langle\hat{N}_{g}\right\rangle$ the maximal QFI attainable with rotations generated by linear combinations of the $\hat{S}_{i}$ is $4 \lambda_{+}^{(x y)}$. The corresponding (normalized) eigenvector

$\mathbf{u}_{\mathrm{opt}}^{(g)}= \pm \frac{1}{\sqrt{2}}\left(\sqrt{1+\sqrt{2} \frac{\alpha^{2}\left\langle\hat{N}_{g}\right\rangle}{\beta^{2} \Delta \hat{N}_{g}}},-\sqrt{1-\sqrt{2} \frac{\alpha^{2}\left\langle\hat{N}_{g}\right\rangle}{\beta^{2} \Delta \hat{N}_{g}}}, 0\right)$

gives the optimal direction of interferometric transformations in the symmetric subspace.

Recall that $\phi_{g h}: \hat{g}^{\dagger} \leftrightarrow i \hat{h}^{\dagger}$ and $\phi_{ \pm}: \hat{a}_{1}^{\dagger} \leftrightarrow \hat{a}_{-1}^{\dagger}$ leave the algebraic relations of the creation and annihilation operators, the initial state $|\psi(0)\rangle$, the Hamiltonian, and thus $|\psi(t)\rangle$ invariant. Hence $\phi_{g h}\left(\hat{N}_{g}\right)=\hat{N}_{h}$ and $\phi_{ \pm}\left(\hat{N}_{+}\right)=\hat{N}_{-}$imply $\left\langle\hat{N}_{h}\right\rangle=$ $\left\langle\hat{N}_{g}\right\rangle, \Delta \hat{N}_{h}=\Delta \hat{N}_{g},\left\langle\hat{N}_{+}\right\rangle=\left\langle\hat{N}_{-}\right\rangle$, and $\Delta \hat{N}_{+}=\Delta \hat{N}_{-}$. Then $\hat{N}_{g}+\hat{N}_{h}=\hat{N}_{+}+\hat{N}_{-}$yields $\left\langle\hat{N}_{+}\right\rangle=\left\langle\hat{N}_{g}\right\rangle$. Using $\hat{D}|\psi(t)\rangle=$ 0 and $|\psi(t)\rangle=|\psi(t)\rangle_{g} \otimes|\psi(t)\rangle_{h}$ we furthermore find that $\Delta \hat{N}_{+}=\Delta \hat{N}_{g} / \sqrt{2}$. Finally, $\phi_{g h}\left(\hat{A}_{x}\right)=-\hat{S}_{y}, \phi_{g h}\left(\hat{A}_{y}\right)=\hat{S}_{x}$, and $\phi_{g h}\left(\hat{A}_{z}\right)=\hat{S}_{z}$ entail that the eigenvalues of $\boldsymbol{\Gamma}_{\hat{\mathbf{A}}}$ coincide with the ones of $\boldsymbol{\Gamma}_{\hat{\mathrm{S}}}$, while

$$
\mathbf{u}_{\mathrm{opt}}^{(h)}=\left(-u_{\mathrm{opt}, y}^{(g)}, u_{\mathrm{opt}, x}^{(g)}, 0\right)
$$

defines, again for $N \gg\left\langle\hat{N}_{ \pm}\right\rangle$, the optimal rotation for phase imprinting within the antisymmetric subspace.
[1] A. D. Cronin, J. Schmiedmayer, and D. E. Pritchard, Optics and interferometry with atoms and molecules, Rev. Mod. Phys. 81, 1051 (2009).

[2] V. Giovannetti, S. Lloyd, and L. Maccone, Quantum Metrology, Phys. Rev. Lett. 96, 010401 (2006).

[3] L. Pezzè and A. Smerzi, Entanglement, Nonlinear Dynamics, and the Heisenberg Limit, Phys. Rev. Lett. 102, 100401 (2009).

[4] P. Hyllus, W. Laskowski, R. Krischek, C. Schwemmer, W. Wieczorek, H. Weinfurter, L. Pezzè, and A. Smerzi, Fisher information and multiparticle entanglement, Phys. Rev. A 85, 022321 (2012); G. Tóth, Multipartite entanglement and highprecision metrology, ibid. 85, 022322 (2012).

[5] L. Pezzè, A. Smerzi, M. K. Oberthaler, R. Schmied, and P. T. Treutlein, Quantum metrology with nonclassical states of atomic ensembles, arXiv:1609.01609.
[6] B. Lücke, M. Scherer, J. Kruse, L. Pezzè, F. Deuretzbacher, P. Hyllus, O. Topic, J. Peise, W. Ertmer, J. Arlt, L. Santos, A. Smerzi, and C. Klempt, Twin matter waves for interferometry beyond the classical limit, Science 334, 773 (2011).

[7] C. Gross, H. Strobel, E. Nicklas, T. Zibold, N. Bar-Gill, G. Kurizki, and M. K. Oberthaler, Atomic homodyne detection of continuous-variable entangled twin-atom states, Nature 480, 219 (2011).

[8] C. D. Hamley, C. S. Gerving, T. M. Hoang, E. M. Bookjans, and M. S. Chapman, Spin-nematic squeezed vacuum in a quantum gas, Nat. Phys. 8, 305 (2012).

[9] B. Lücke, J. Peise, G. Vitagliano, J. Arlt, L. Santos, G. Tóth, and C. Klempt, Detecting Multiparticle Entanglement of Dicke States, Phys. Rev. Lett. 112, 155304 (2014). 
[10] J. Peise, I. Kruse, K. Lange, B. Lücke, L. Pezzè, J. Arlt, W. Ertmer, K. Hammerer, L. Santos, A. Smerzi, and C. Klempt, Satisfying the Einstein-Podolsky-Rosen criterion with massive particles, Nat. Commun. 6, 8984 (2015).

[11] I. Kruse, K. Lange, J. Peise, B. Lücke, L. Pezzè, J. Arlt, W. Ertmer, C. Lisdat, L. Santos, A. Smerzi, and C. Klempt, Improvement of an Atomic Clock Using Squeezed Vacuum, Phys. Rev. Lett. 117, 143004 (2016).

[12] D. Linnemann, H. Strobel, W. Muessel, J. Schulz, R. J. Lewis-Swan, K. V. Kheruntsyan, and M. K. Oberthaler, Quantum-Enhanced Sensing Based on Time Reversal of Nonlinear Dynamics, Phys. Rev. Lett. 117, 013001 (2016); D. Linnemann, J. Schulz, W. Muessel, P. Kunkel, M. Prüfer, A. Frölian, H. Strobel, and M. K. Oberthaler, Active SU(1, 1) atom interferometry, Quantum Science and Technology 2, 044009 (2017).

[13] Z. Zhang and L.-M. Duan, Generation of Massive Entanglement through an Adiabatic Quantum Phase Transition in a Spinor Condensate, Phys. Rev. Lett. 111, 180401 (2013).

[14] L.-N. Wu and L. You, Using the ground state of an antiferromagnetic spin-1 atomic condensate for Heisenberg-limited metrology, Phys. Rev. A 93, 033608 (2016).

[15] D. Kajtoch, K. Pawlowski, and E. Witkowska, Metrologically useful states of spin-1 Bose condensates with macroscopic magnetization, Phys. Rev. A 97, 023616 (2018).

[16] D. M. Stamper-Kurn and M. Ueda, Spinor Bose gases: Symmetries, magnetism, and quantum dynamics, Rev. Mod. Phys. 85, 1191 (2013).

[17] X.-Y. Luo, Y.-Q. Zou, L.-N. Wu, Q. Liu, M.-F. Han, M. K. Tey, and L. You, Deterministic entanglement generation from driving through quantum phase transitions, Science 355, 620 (2017).

[18] T. M. Hoang, H. M. Bharath, M. J. Boguslawski, M. Anquez, B. A. Robbins, and M. S. Chapman, Adiabatic quenches and characterization of amplitude excitations in a continuous quantum phase transition, Proc. Natl. Acad. Sci. U. S. A. 113, 9475 (2016).

[19] A. Vinit and C. Raman, Precise measurements on a quantum phase transition in antiferromagnetic spinor Bose-Einstein condensates, Phys. Rev. A 95, 011603(R) (2017).

[20] L. Pezzè, M. Gessner, P. Feldmann, C. Klempt, L. Santos, and A. Smerzi, Heralded Generation of Macroscopic Superposition States in a Spinor Bose-Einstein Condensate, arXiv:1712.03864.
[21] L. Pezzè and A. Smerzi, in Atom Interferometry, Proceedings of the International School of Physics Enrico Fermi, Vol. 188, edited by G. M. Tino and M. A. Kasevich (IOS, Amsterdam, 2014), pp. 691-741; arXiv:1411.5164.

[22] S. L. Braunstein and C. M. Caves, Statistical Distance and the Geometry of Quantum States, Phys. Rev. Lett. 72, 3439 (1994).

[23] C. W. Helstrom, Quantum Detection and Estimation Theory (Academic, New York, 1976).

[24] M. Gessner, L. Pezzè, and A. Smerzi, Efficient entanglement criteria for discrete, continuous, and hybrid variables, Phys. Rev. A 94, 020101(R) (2016).

[25] C. K. Law, H. Pu, and N. P. Bigelow, Quantum Spins Mixing in Spinor Bose-Einstein Condensates, Phys. Rev. Lett. 81, 5257 (1998).

[26] Y. Kawaguchi and M. Ueda, Spinor Bose-Einstein condensates, Phys. Rep. 520, 253 (2012).

[27] We assume $\lambda=\frac{4 \pi \hbar^{2}\left(a_{2}-a_{0}\right)}{3 m} \int d^{3} r|\phi(\mathbf{r})|^{4}$, where $\phi(\mathbf{r})$ is the condensate (Gross-Pitaevskii) wave function normalized such that $\int d^{3} r|\phi(\mathbf{r})|^{2}=1, m$ is the atomic mass and $a_{G}$ are the scattering lengths for $s$-wave collisions in the $G=0,2$ allowed channels.

[28] M. J. Holland and K. Burnett, Interferometric Detection of Optical Phase Shifts at the Heisenberg Limit, Phys. Rev. Lett. 71, 1355 (1993).

[29] L.-M. Duan, A. Sørensen, J. I. Cirac, and P. Zoller, Squeezing and Entanglement of Atomic Beams, Phys. Rev. Lett. 85, 3991 (2000).

[30] H. Pu and P. Meystre, Creating Macroscopic Atomic EinsteinPodolsky-Rosen States from Bose-Einstein Condensates, Phys. Rev. Lett. 85, 3987 (2000).

[31] L.-M. Duan, J. I. Cirac, and P. Zoller, Quantum entanglement in spinor Bose-Einstein condensates, Phys. Rev. A 65, 033619 (2002).

[32] M. Gabbrielli, L. Pezzè, and A. Smerzi, Spin-Mixing Interferometry with Bose-Einstein Condensates, Phys. Rev. Lett. 115, 163002 (2015).

[33] S. S. Szigeti, R. J. Lewis-Swan, and S. A. Haine, Pumped-Up SU(1, 1) Interferometry, Phys. Rev. Lett. 118, 150401 (2017).

[34] Y.-Q. Zou, L.-N. Wu, Q. Liu, X.-Y. Luo, S.-F. Guo, J.-H. Cao, M. K. Tey, and L. You, Beating the classical precision limit with spin-1 Dicke state of more than 10000 atoms, arXiv:1802.10288.

[35] N. E. Bogoliubov and D. V. Shirkov, Introduction to the Theory of Quantized Fields (Wiley-Interscience, New York, 1959).

[36] D. R. Truax, Baker-Campbell-Hausdorff relations and unitarity of SU(2) and SU(1, 1) squeeze operators, Phys. Rev. D 31, 1988 (1985). 